\title{
Effect of the secondary process on mass point vibration velocity propagation in magneto-acoustic tomography and magneto-acousto-electrical tomography
}

\author{
Zhishen Sun ${ }^{\mathrm{a}, \mathrm{b}}$, Guoqiang Liu ${ }^{\mathrm{a}, *}$, Liang Guo ${ }^{\mathrm{a}, \mathrm{b}, \mathrm{c}}$, Hui Xia ${ }^{\mathrm{a}}$ and Xinli Wang ${ }^{\mathrm{a}}$ \\ ${ }^{a}$ Institute of Electrical Engineering, Chinese Academy of Sciences, Beijing, China \\ ${ }^{\mathrm{b}}$ University of Chinese Academy of Sciences, Beijing, China \\ ${ }^{\mathrm{c}}$ College of Control Theory and Engineering, China University of Petroleum, Qingdao, Shandong, \\ China
}

\begin{abstract}
.
BACKGROUND: As two of the new biological electrical impedance tomography (EIT), magneto-acoustic tomography (MAT) and magneto-acousto-electrical tomography (MAET) achieve both the high contrast property of EIT and the high spatial resolution property of sonography through combining EIT and sonography. As both MAT and MAET contain a uniform magnetic field, vibration and electrical current density, there is a secondary process both in MAT and in MAET, which is MAET and MAT respectively.

OBJECTIVE: To analyze the effect of the secondary process on mass point vibration velocity (MPVV) propagation in MAT and MAET.

METHODS: By analyzing the total force to the sample, the wave equations of MPVV in MAT and MAET - when the secondary processes were considered - were derived. The expression of the attenuation constant in the wave number was derived in the case that the mass point vibration velocity propagates in the form of cylindrical wave and plane wave. Attenuations of propagation of the MPVV in several samples were quantified.

RESULTS: Attenuations of the MPVV after propagating for $1 \mathrm{~mm}$ in copper or aluminum foil, and for $5 \mathrm{~cm}$ in gel phantom or biological soft tissue were less than $1 \%$.

CONCLUSION: Attenuations of the MPVV in MAT and MAET due to the secondary processes are relatively minor, and effects of the secondary processes on MPVV propagation in MAT and MAET can be ignored.
\end{abstract}

Keywords: Magneto-acoustic tomography, magneto-acousto-electrical tomography, wave equation of mass point vibration velocity, linear damping

\section{Introduction}

Magneto-acoustic tomography (MAT) [1] and magneto-acousto-electrical tomography (MAET) [5] - which have rapidly developed in recent years - combine the merits of high contrast of electrical impedance tomography (EIT) and high spatial resolution of sonography. In MAT, the object sample

\footnotetext{
${ }^{*}$ Corresponding author: Guoqiang Liu, Institute of Electrical Engineering, Chinese Academy of Sciences, Beijing 100190, China. Tel.: +86 10 82547190; Fax: +8610 82547189; E-mail: gqliu@ mail.iee.ac.cn.
}

0928-7329/16/\$35.00 (c) 2016 - IOS Press and the authors. All rights reserved This article is published online with Open Access and distributed under the terms of the Creative Commons Attribution NonCommercial License. 


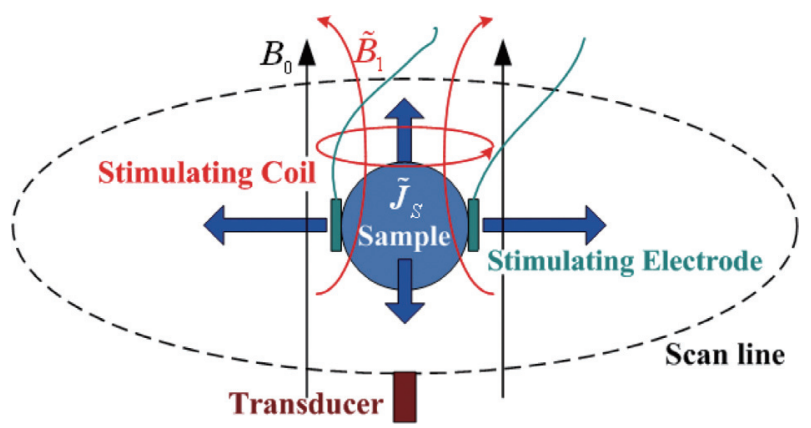

Fig. 1. Schematic diagram of magneto-acoustic tomography model.

is placed in a uniform magnetic field and electrical current density inside the sample was generated either by means of induction using a stimulating coil or by injection through a pair of stimulating electrodes. The electrical current density inside the sample couples with the uniform magnetic field, and the Lorentz force is generated and acts on the sample, which causes the sample to vibrate. As the vibration propagates, acoustic pressures around the sample in different directions are collected using an ultrasound transducer, and then using these acoustic pressures, the relative conductivity of the sample is obtained through inverse problem algorithm. Xu developed magneto-acoustic tomography with magnetic induction (MAT-MI) [2], and Liu developed magneto-acoustic tomography with current injection (MAT-CI) [3]. In MAET, the object sample is also placed in a uniform magnetic field. Ultrasound from the transducer is used to cause the sample to vibrate. As the sample moves, the positive and negative ions in the sample also move, but due to the Lorentz force acting on the moving ions, the positive and negative ions deviate from the direction of the movement of the sample and depart for the opposite directions, resulting in voltage difference and electrical current density being generated in the sample [4]. The voltage difference can be measured using electrodes and the electrical current density through a coil. Xu developed magneto-acousto-electrical tomography with electrode detection [5,6]. Guo developed magneto-acousto-electrical tomography with coil detection (MAET-MI) [7].

In MAT, the object sample movement in the uniform magnetic field generates voltage difference and electrical current density, so MAET (the secondary process in MAT) exists; similarly, in MAET, the electrical current density in the sample coupled with the uniform magnetic field generates the Lorentz force that acts on the sample and causes the sample to vibrate, indicating the existence of MAT (the secondary process in MAET). Until now all research on MAT and MAET has ignored the secondary processes, but no explanation was given [8-13]. In this study, wave equations of MPVV in MAT and MAET are derived when the secondary processes are taken into account, and it's found that the secondary process can lead to linear damping to MPVV propagation. Besides, the expression of the attenuation constant is derived when MPVV propagates in the form of cylindrical or plane wave. Finally, the attenuations induced by linear damping in copper foil, aluminum foil, gel phantom and biological soft tissue - are calculated when the ultrasound in the range of $0.5 \mathrm{MHz}$ and $20 \mathrm{MHz}$ is used.

\section{Theory}

\subsection{Influence of the secondary process as MAET to MAT}

In MAT, as shown in Fig. 1, the object sample is placed in the uniform magnetic field $\boldsymbol{B}_{0}$ and the electrical current density $\tilde{\boldsymbol{J}}_{1}$ is generated either through injection using a pair of stimulating electrodes or by 
means of induction using a coil. The electrical current density works together with the uniform magnetic field and the first Lorentz force $\tilde{f}_{1}$ acting on object sample is generated, which results in vibration of the object sample. And an ultrasound transducer is placed around the sample in different directions each time to collect the acoustic pressure. The secondary process in MAT is MAET, in which the movement of the sample in uniform magnetic field produces the secondary electrical current density $\tilde{\boldsymbol{J}}_{2}$, and furthermore, the secondary electrical current density coupled with uniform magnetic field generates the secondary Lorentz force $\tilde{\boldsymbol{f}}_{2}$. The combination of the first and secondary Lorentz forces is to be the total force to the sample. The object sample is assumed to be acoustically homogenous with constant equilibrium density $\rho_{0}$ and acoustic velocity $c_{s}$, and wave equation of MPVV $\tilde{\boldsymbol{v}}$ is obtained as Eq. (1) [14].

$$
\rho_{0} \frac{\partial^{2} \tilde{\boldsymbol{v}}}{\partial t^{2}}-\rho_{0} c_{s}^{2} \nabla \nabla \cdot \tilde{\boldsymbol{v}}=\frac{\partial \tilde{\boldsymbol{f}}}{\partial t}
$$

The total magnetic field contains the uniform magnetic field, the stimulating magnetic field (if stimulating coil is used) and the secondary magnetic field. But, as the uniform magnetic flux density is far higher than that of the stimulating magnetic flux density and the secondary magnetic flux density, only the uniform magnetic flux density is included when calculating the total magnetic flux density. Then the total force acting on the sample is gotten in the form of Eq. (2).

$$
\tilde{\boldsymbol{f}} \approx\left(\sigma \tilde{\boldsymbol{E}}_{1}+\sigma \tilde{\boldsymbol{v}} \times \tilde{\boldsymbol{B}}_{0}\right) \times \tilde{\boldsymbol{B}}_{0}
$$

Using Eqs (1) and (2), wave equation of MPVV is obtained as Eq. (3) when the MAET secondary effect is included.

$$
\nabla \nabla \cdot \tilde{\boldsymbol{v}}-\frac{1}{c_{s}^{2}} \frac{\partial^{2} \tilde{\boldsymbol{v}}}{\partial t^{2}}-\frac{B_{0}^{2} \sigma}{\rho_{0} c_{s}^{2}} \frac{\partial \tilde{\boldsymbol{v}}}{\partial t}=-\frac{\sigma}{\rho_{0} c_{s}^{2}} \boldsymbol{E}_{1} \times \boldsymbol{B}_{0} g^{\prime}(t)
$$

If the MAET secondary effect is ignored $-\tilde{\boldsymbol{J}}_{2}$ is neglected as well, then the total force reduces to the form of Eq. (4).

$$
\tilde{\boldsymbol{f}} \approx \sigma \tilde{\boldsymbol{E}}_{1} \times \tilde{\boldsymbol{B}}_{0}
$$

Using Eqs (1) and (4), wave equation of MPVV is obtained as Eq. (5) if the MAET secondary effect is excluded.

$$
\nabla \nabla \cdot \tilde{\boldsymbol{v}}-\frac{1}{c_{s}^{2}} \frac{\partial^{2} \tilde{\boldsymbol{v}}}{\partial t^{2}}=-\frac{\sigma}{\rho_{0} c_{s}^{2}} \boldsymbol{E}_{1} \times \tilde{\boldsymbol{B}}_{0} g^{\prime}(t)
$$

Compared with Eq. (5), Eq. (3) contains the first order derivative of MPVV $\tilde{\boldsymbol{v}}$ with respect to time. As is known that the first-order derivative term in wave equation with respect to time corresponds to linear damping, the MAET secondary effect in MAT brings about attenuation to MPVV propagation.

\subsection{Influence of the secondary process as MAT to MAET}

In MAET shown in Fig. 2, an ultrasound transducer placed around the object sample in different directions transmits ultrasound wave towards the object sample, and the object sample in the uniform magnetic field $\boldsymbol{B}_{0}$ vibrates as the ultrasound passes by. As in the direction of vibration which both the positive and negative ions move along, there is no electrical current. But in the direction perpendicular 


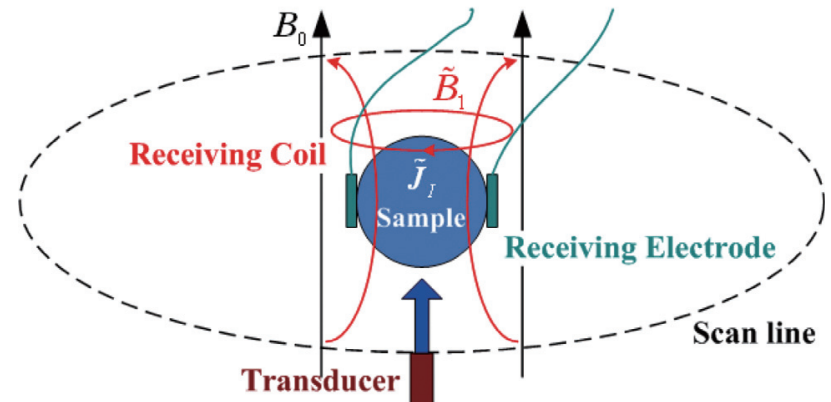

Fig. 2. Schematic diagram of magneto-acousto-electrical tomography model.

both to the vibration direction and to the uniform magnetic field direction, the positive and negative ions deviate, due to the Lorentz force, for the opposite directions and electrical current density $\tilde{J}_{1}$ is generated. The secondary process in MAET is MAT, in which the electrical current density coupled with the uniform magnetic field generates a Lorentz force on the sample, which causes the sample to vibrate. Assuming the ultrasound transducer be point acoustic source, wave equation of the MPVV in MAET can be written as Eq. (6) when the secondary MAT effect is considered [14].

$$
\nabla \nabla \cdot \tilde{\boldsymbol{v}}-\frac{1}{c_{s}^{2}} \frac{\partial^{2} \tilde{\boldsymbol{v}}}{\partial t^{2}}=-\frac{1}{\rho_{0} c_{s}^{2}} \frac{\partial \tilde{\boldsymbol{f}}}{\partial t}+\frac{1}{\rho_{0}} \nabla \delta\left(\boldsymbol{r}-\boldsymbol{r}^{\prime}\right) u(t)
$$

The induced electrical current density from $\tilde{\boldsymbol{J}}_{1}$ is ignored when calculating the total electrical current density $\tilde{\boldsymbol{J}}$ since it is far smaller than $\tilde{\boldsymbol{J}}_{1}$. And the total force on the sample becomes the right hand side of Eq. (7).

$$
\tilde{\boldsymbol{f}} \approx-B_{0}^{2} \sigma \tilde{\boldsymbol{v}}
$$

Combining Eqs (6) and (7), wave equation of MPVV in MAET arrives to Eq. (8) in the case of the secondary MAT effect being included.

$$
\nabla \nabla \cdot \tilde{\boldsymbol{v}}-\frac{1}{c_{s}^{2}} \frac{\partial^{2} \tilde{\boldsymbol{v}}}{\partial t^{2}}-\frac{B_{0}^{2} \sigma}{\rho_{0} c_{s}^{2}} \frac{\partial \tilde{\boldsymbol{v}}}{\partial t}=\frac{1}{\rho_{0}} \nabla \delta\left(\boldsymbol{r}-\boldsymbol{r}^{\prime}\right) u(t)
$$

When the secondary MAT effect is not taken into account - or equivalently, $\tilde{f}$ equals zero here, the wave equation of the MPVV in MAET reduces to Eq. (9).

$$
\nabla \nabla \cdot \tilde{\boldsymbol{v}}-\frac{1}{c_{s}^{2}} \frac{\partial^{2} \tilde{v}}{\partial t^{2}}=\frac{1}{\rho_{0}} \nabla \delta\left(\boldsymbol{r}-\boldsymbol{r}^{\prime}\right) u(t)
$$

Comparing Eqs (8) and (9), like the result in Section 2.1, one additional term in the form of the first-order derivative with respect to time also exists in the wave equation of MPVV in MAET when the secondary MAT effect is accounted for, which means the MPVV propagation in MAET is also linear damping. And the MAT secondary effect brings attenuation to MPVV propagation in MAET. 


\subsection{Effect of linear damping on MPVV propagation}

In order to analyze the effect of linear damping on MPVV propagation independently, only the corresponding homogeneous wave equation is analyzed. It can be seen that the homogeneous wave equations of Eqs (3) and (8) are the same; and Eqs (5) and (9) also have the same homogeneous wave equations. So, only one of the two groups of homogeneous wave equations is needed for analysis, i.e. Eqs (10) and (11).

$$
\begin{aligned}
& \nabla \nabla \cdot \tilde{\boldsymbol{v}}-\frac{1}{c_{s}^{2}} \frac{\partial^{2} \tilde{\boldsymbol{v}}}{\partial t^{2}}-\frac{B_{0}^{2} \sigma}{\rho_{0} c_{s}^{2}} \frac{\partial \tilde{\boldsymbol{v}}}{\partial t}=0 \\
& \nabla \nabla \cdot \tilde{\boldsymbol{v}}-\frac{1}{c_{s}^{2}} \frac{\partial^{2} \tilde{\boldsymbol{v}}}{\partial t^{2}}=0
\end{aligned}
$$

The Fourier transforms of Eqs (10) and (11) are given by Eqs (12) and (13), respectively, where $\boldsymbol{V}$ is the Fourier transform of $\tilde{\boldsymbol{v}}$ and $\omega^{2} / c_{s}^{2}$ is the square of wave number, which is real and positive when no linear damping exists.

$$
\begin{aligned}
& \nabla \nabla \cdot \boldsymbol{V}+\left(\frac{\omega^{2}}{c_{s}^{2}}-i \omega \frac{\left|\tilde{\boldsymbol{B}}_{0}\right|^{2} \sigma}{\rho_{0} c_{s}^{2}}\right) \boldsymbol{V}=0 \\
& \nabla \nabla \cdot \boldsymbol{V}+\frac{\omega^{2}}{c_{s}^{2}} \boldsymbol{V}=0
\end{aligned}
$$

As is shown in Eq. (12), the square of wave number is no longer real and becomes complex in the case that linear damping exists. The propagation constant, $\gamma$ in the form of Eq. (14), is used.

$$
\gamma=i k=i \sqrt{\frac{\omega^{2}}{c_{s}^{2}}-i \omega \frac{B_{0}^{2} \sigma}{\rho_{0} c_{s}^{2}}}
$$

Since $\gamma$ is complex, it can be written as Eq. (15), where $\alpha$ and $\beta$ are called the attenuation constant and the phase constant, respectively.

$$
\begin{aligned}
& \gamma=\alpha+i \beta \\
& \frac{B_{0}^{4} \sigma^{2}}{\rho_{0}^{2} \omega^{2}} \ll 1 \\
& \left\{\begin{array}{l}
\alpha \approx \frac{1}{2} \frac{B_{0}^{2} \sigma}{\rho_{0} c_{s}} \\
\beta=\frac{\omega}{\sqrt{2} c_{s}}\left[\sqrt{1+\frac{B_{0}^{4} \sigma^{2}}{\rho_{0}^{2} \omega^{2}}}+1\right]^{\frac{1}{2}}
\end{array}\right.
\end{aligned}
$$

When uniform magnetic flux density, conductivity and angular frequency satisfy inequality (16), combining Eqs (14) and (15), analytical expression of $\alpha$ and $\beta$ can be derived, as is shown in Eq. (17).

In MAT, as the uniform magnetic field $\boldsymbol{B}_{0}$ and the stimulating magnetic field $\tilde{\boldsymbol{B}}_{1}$ (MAT-MI is used) are in the direction of $e_{z}$, the electrical current density $-\boldsymbol{e}_{\phi}$ and the Lorentz force $-\boldsymbol{e}_{\rho}$, MPVV can be 
Table 1

Attenuation in different samples

\begin{tabular}{cccccccc}
\hline & $\rho_{0}\left(\mathrm{~kg} / \mathrm{m}^{3}\right)$ & $c_{s}(\mathrm{~m} / \mathrm{s})$ & $\sigma(S / \mathrm{m})$ & $\begin{array}{c}B_{0}^{4} \sigma^{2} /\left(\rho_{0}^{2} \omega^{2}\right) \\
f=2.25 \mathrm{MHz}\end{array}$ & & \multicolumn{2}{c}{$e^{-\alpha s}$} \\
\cline { 5 - 7 } & & & & $f=1 \mathrm{~mm}$ & $s=50 \mathrm{~mm}$ \\
\hline $\mathrm{A}$ & $8.93 \times 10^{3}$ & 4674 & $5.80 \times 10^{7}$ & $2.1 \times 10^{-7}$ & -0.69 & 0.999 & - \\
$\mathrm{B}$ & $2.70 \times 10^{3}$ & 6305 & $3.54 \times 10^{7}$ & $8.6 \times 10^{-7}$ & -1.04 & 0.999 & - \\
$\mathrm{C}$ & $10^{3}$ & 1500 & 20 & $2.0 \times 10^{-18}$ & $-6.7 \times 10^{-6}$ & - & 1 \\
$\mathrm{D}$ & $1.02 \times 10^{3}$ & 1500 & 0.1 & $4.8 \times 10^{-23}$ & $-3.3 \times 10^{-8}$ & - & 1 \\
\hline
\end{tabular}

A: Copper foil; B: Aluminum foil; C: Gel phantom; D: Biological soft tissue.

approximated as propagating in $\boldsymbol{e}_{\rho}$. And in MAET, as the uniform magnetic field $B_{0}$ is in the direction of $e_{z}$, the ultrasound $-\boldsymbol{e}_{x}$, the electrical current density $\boldsymbol{e}_{y}$ and the Lorentz force $\boldsymbol{e}_{x}$, the MPVV can be approximated as propagating in the direction of $e_{x}$. Both in MAT and in MAET, $\tilde{\boldsymbol{v}}$ can be written in the form of Eq. (18). In MAT, $s$ is $\rho$ whereas in MAET, $s$ is $x$. And when $k$ is in the form of Eq. (14), $\tilde{\boldsymbol{v}}$ becomes Eq. (19).

$$
\begin{aligned}
& \tilde{\boldsymbol{v}}=A_{0} e^{i(\omega t-k s)} \boldsymbol{e}_{s} \\
& \tilde{\boldsymbol{v}}=A_{0} e^{-\alpha s} e^{i(\omega t-\beta s)} \boldsymbol{e}_{s}
\end{aligned}
$$

When there is no linear damping, $k$ is real and positive and $\tilde{\boldsymbol{v}}$ propagates without attenuation; and when linear damping exists, $\tilde{v}$ attenuates with the attenuation constant correlating with uniform magnetic flux density, the conductivity, constant equilibrium density and the acoustic speed of the sample as it propagates.

\section{Calculation and discussion}

Using Eq. (17), attenuations of MPVV propagation in four commonly used materials - copper foil, aluminum foil, gel phantom and biological soft tissue - are calculated. Uniform magnetic flux density of 1T and system frequency of $2.25 \mathrm{MHz}$ are assumed, and the values of left hand side of Eq. (16) are calculated and listed in Table 1. These values are far less than 1 and inequality Eq. (16) is satisfied. What's more, attenuations of the MPVV after propagating for $1 \mathrm{~mm}$ in copper or aluminum foil, and for $5 \mathrm{~cm}$ in gel phantom or biological soft tissue are calculated and listed in Table 1, but the results showed that these attenuations are less than $1 \%$.

\section{Conclusion}

In this work, wave equations of the MPVV in MAT and MAET are given when the secondary processes are taken into account. Both wave equations contain the first-order time derivative term, which corresponds to linear damping in wave propagation. Moreover, the analytical expression of the attenuation constant is derived when MPVV propagates as cylindrical wave or plane wave. Furthermore, calculation of the attenuations of the MPVV after propagating for $1 \mathrm{~mm}$ in copper or aluminum foil and for $5 \mathrm{~cm}$ in gel phantom or biological soft tissue were done. These attenuations are too minor to be none. So, effects on MPVV propagation of the secondary processes in MAT and MAET can be ignored. 


\section{Acknowledgements}

This work was supported in part by the National Natural Science Foundation of China under Grants 51137004,61427806 and 51477161.

\section{References}

[1] Towe BC. Islam MR. A magneto-acoustic method for the noninvasive measurement of bioelectric currents[J]. Biomedical Engineering, IEEE Transactions on, 1988, 35(10): 892-894.

[2] $\mathrm{Xu} \mathrm{Y.} \mathrm{He} \mathrm{B.} \mathrm{Magnetoacoustic} \mathrm{tomography} \mathrm{with} \mathrm{magnetic} \mathrm{induction} \mathrm{(MAT-MI)[J].} \mathrm{Physics} \mathrm{in} \mathrm{Medicine} \mathrm{and} \mathrm{Biology,}$ 2005, 50(21): 5175 .

[3] Liu GQ. Huang X. Xia H. et al. Magnetoacoustic tomography with current injection[J]. Chinese Science Bulletin, 2013 , 58(30): 3600-3606.

[4] Wen H. Shah J. Balaban RS. Hall effect imaging[J]. Biomedical Engineering, IEEE Transactions on, 1998, 45(1): 119124.

[5] Xu Y. Haider S. Hrbek A. Magneto-acousto-electrical tomography: A new imaging modality for electrical impedance[C]. 13th International Conference on Electrical Bioimpedance and the 8th Conference on Electrical Impedance Tomography. Springer Berlin Heidelberg, 2007: 292-295.

[6] Haider S. Hrbek A. Xu Y. Magneto-acousto-electrical tomography: A potential method for imaging current density and electrical impedance[J]. Physiological Measurement, 2008, 29(6): S41-50.

[7] Guo L. Liu G. Xia H. Magneto-Acousto-Electrical Tomography with Magnetic Induction for Conductivity Reconstruction[J]. IEEE Transactions on Bio-medical Engineering, 2014

[8] Wang H. The algorithm of magnetoacoustic tomography with magnetic induction, Master's thesis, Graduate School of Chinese Academy of Sciences, 2008.

[9] Li X. A study on magnetoacoustic tomography with magnetic induction(MAT-MI) for imaging elctrical impedance of biological tissue, Ph.D.Dissertation, Zhengjiang University, 2009.

[10] Li X. Magnetoacoustic Tomography with Magnetic Induction for Electrical Conductivity Imaging of Biological Tissue, Ph.D. Dissertation, University of Minnesota, 2010.

[11] He W. A study on sound source mechanism and algorithm of magnetoacoustic tomography with mag-netic induction, Master's thesis, Graduate School of Chinese Academy of Sciences, 2011.

[12] Mariappan L. Magnetoacoustic tomography with magnetic induction for electrical conductivity based tissue imaging, Ph.D. Dissertation University of Minnesota, 2014.

[13] Zeng X. Research for Magneto-Acousto-Electrical Tomography, Master's thesis, Graduate School of Chinese Academy of Sciences, 2011.

[14] Zhang H. Theoretical Acoustics (Revised Edition), Higher Education Press, China, 2012, pp. 176-183. 